\title{
Recent advances in the diagnosis and management of giant cell arteritis
}

\author{
Naomi Serling-Boyd, John H. Stone \\ Division of Rheumatology, Allergy and Immunology, Massachusetts General Hospital, Boston, \\ Massachusetts, USA
}

\begin{abstract}
Purpose of review-Giant cell arteritis (GCA) has classically been diagnosed by temporal artery biopsy and treated with high-dose, long-term glucocorticoid therapy. Noninvasive imaging increasingly is employed for diagnostic purposes, but further studies are needed to determine the role of imaging in monitoring longitudinal disease activity. Glucocorticoid-sparing therapy mitigates the numerous adverse effects of glucocorticoids. This review addresses new developments in these areas.
\end{abstract}

Recent findings-For diagnosis, when performed at a center with expertise in its use, temporal artery ultrasound has an estimated sensitivity and specificity of 78 and $79 \%$, respectively. State-ofthe-art time-of-flight positron emission tomography/computed tomography (PET/CT) has an estimated sensitivity and specificity of 71 and $91 \%$, respectively. The sensitivities of both imaging modalities decrease following glucocorticoid administration. Tocilizumab is an effective glucocorticoid-sparing therapy, demonstrating sustained glucocorticoid-free remission in $56 \%$ of patients receiving weekly tocilizumab compared with $18 \%$ of patients receiving a 52-week prednisone taper. The traditional acute phase reactants are of no value in patients treated with interleukin-6 receptor (IL6-R) blockade, and thus, the development of new biomarkers is an important priority in the field.

Summary-Noninvasive imaging techniques are increasingly used in the absence of temporal artery biopsy to confirm diagnostic suspicions of GCA. Tocilizumab reduces the cumulative glucocorticoid exposure and increases the rate of sustained remission. Ongoing efforts are directed towards new methods to identify disease flares.

\section{Keywords}

giant cell arteritis; large vessel vasculitis; tocilizumab

\section{INTRODUCTION}

Giant cell arteritis (GCA) is a form of large vessel vasculitis. It affects patients older than 50 years of age and is approximately three times more common among women than men. Symptoms include headache, jaw claudication, scalp tenderness, fevers, weight loss, and

Correspondence to John H. Stone, Yawkey Center for Outpatient Care (MGH), 32 Fruit Street, Boston, MA, 02114, USA. Tel: +1617 726 7938; jhstone@mgh.harvard.edu. 
symptoms of polymyalgia rheumatica (PMR). In addition, ocular symptoms, such as transient or permanent vision loss or stroke may occur. No diagnostic laboratory test exists for this disease. Temporal artery biopsy has traditionally been considered the 'gold standard' for diagnosis, though the sensitivity of biopsy may be as low as 50\%. More recently, imaging modalities have been employed with increasing frequency to establish the diagnosis without a positive temporal artery biopsy. The prompt institution of treatment is important once the diagnosis of GCA is considered as failure to initiate therapy in a timely manner may lead to unfortunate clinical outcomes, such as blindness. Although glucocorticoids are effective at controlling GCA and preventing vision loss, they can be poorly tolerated, and multiple attempts have been made through the years to identify effective glucocorticoidsparing agents. Most recently, tocilizumab, an interleukin-6 (IL-6) receptor alpha inhibitor, has been identified as an effective agent. This review will focus on recent updates in the diagnosis and management of GCA.

\section{ESTABLISHING THE DIAGNOSIS: ULTRASOUND OF THE TEMPORAL ARTERY AND OTHER ARTERIES}

The European League Against Rheumatism (EULAR) recommendations for imaging in large vessel vasculitis regard temporal artery ultrasound as the first-line imaging study for patients suspected of having GCA [1--]. A positive ultrasound study is considered adequate to establish the diagnosis of GCA in the absence of a temporal artery biopsy if suspicion for the diagnosis is high. Findings consistent with mural inflammation and GCA include a hypoechoic 'halo sign' (Fig. 1), occlusion, or stenosis of the temporal artery. The test characteristics of the finding of a hypoechoic halo are estimated to be $68 \%$ for sensitivity and $81 \%$ for specificity. These estimates increase to 78 and $79 \%$, respectively, if any ultrasonographic abnormality is considered (Table 1) [2-m]. Of note, most studies examining temporal artery ultrasound were performed in academic medical centers with expertise in ultrasound imaging of the temporal arteries. Whether ultrasound should be used to guide the temporal artery biopsy site is unclear. One study randomized patients with suspected GCA to ultrasound-guided temporal artery biopsy or standard temporal artery biopsy, in which the ophthalmologist attempted to select a segment of temporal artery that was either tender or nodular on examination when possible and found no improvement in the sensitivity of temporal artery biopsy in the ultrasound-guided group [3]. There has been some suggestion that a 'directional' biopsy (i.e. biopsy ipsilateral to where the halo sign was present) may increase the sensitivity in patients with a unilateral halo sign [4]. The sensitivity of ultrasound decreases following the use of glucocorticoids, from $92 \%$ after $0-1$ days of glucocorticoid treatment to $80 \%$ after 2-4 days of glucocorticoids, and to $50 \%$ after more than 4 days of glucocorticoids [5].

Compared with the change in sensitivity of temporal artery biopsy with glucocorticoid treatment, the sensitivity of the halo sign on ultrasound may decrease more rapidly, whereas the histopathologic changes on biopsy subside more slowly. An early study showed no decrease in positive temporal artery biopsies in patients treated with glucocorticoids prior to biopsy, though more recent work has shown a decrease in sensitivity over time. One study showed that temporal artery biopsy was positive in $78 \%$ of patients treated with less than 2 
weeks of glucocorticoids prior to biopsy, compared with $65 \%$ treated for 2-4 weeks, and $40 \%$ treated for more than 4 weeks [6,7]. Another study evaluated patients with GCA and assigned them to repeat temporal artery biopsy at varying time points and found that after 3 months, $70 \%$ were still positive; after 6 months, $75 \%$ were still positive; after 9 months, $44 \%$ were still positive; and after 12 months, $44 \%$ were still positive [8].

Of note, US has very limited value in evaluating the thoracic aorta and should not be used as a reliable method of detecting aortitis or other large vessel involvement. Whether ultrasound is helpful in confirming or excluding a flare of GCA in a patient with an established diagnosis is still under investigation [ $1 \mathbf{m}]$. Overall, ultrasound is an excellent diagnostic tool, though is subject to operator dependence, and thus it is important that it be utilized in centers where those performing and interpreting the studies are well versed in its use for the assessment of giant cell arteritis. Its sensitivity and specificity may further vary outside of academic centers, and further studies evaluating its utility in a variety of clinical practices will be helpful moving forward.

\section{ESTABLISHING THE DIAGNOSIS: PET/COMPUTED TOMOGRAPHY AND MRI}

EULAR has developed a set of guidelines for imaging in large vessel vasculitis. Positron emission tomography/computed tomography (PET/CT) is not recommended as part of the first-line imaging approach to diagnosis. PET/CT, however, is particularly helpful in assessing aortitis, which is detected most commonly in the ascending aorta. Data are increasingly emerging that PET/CT has a reasonable sensitivity in identifying vasculitis not only in the extracranial large blood vessels but also in the cranial vessels. A recently developed method that employs $1 \mathrm{~mm}$ tomographic cuts, for example, improves the ability of PET/CT to evaluate the temporal arteries [9-].

A recent study evaluated 64 patients who underwent time of flight PET/CT. Compared with temporal artery biopsy, sensitivity was $92 \%$ and specificity was $85 \%$ for diagnosing temporal arteritis. Compared with clinical diagnosis, the sensitivity of this PET/CT procedure was $71 \%$ and its specificity was $91 \%$. PET/CT also had a negative predictive value of $98 \%$, demonstrating its great utility in excluding GCA in lower risk patients. Aortitis is detected on PET/CT in almost half of patients with a positive temporal artery biopsy, and among patients with large vessel vasculitis detected on PET/CT, an average of four vascular territories are involved (Fig. 2) [9-,10].

Disadvantages of using PET/CT include high cost, radiation exposure, and lack of widespread availability of the newer generation imaging [9-]. In addition, as is true for other imaging modalities, fluorodeoxyglucose (FDG) uptake, and consequently the test's sensitivity decrease significantly after glucocorticoid exposure. It is recommended, therefore, that PET/CT be performed within $72 \mathrm{~h}$ of initiating glucocorticoids for the most accurate diagnosis $[11-]$. An additional potential shortcoming is that many patients maintain some degree of FDG uptake in the large vessels even after treatment; thus, its role in monitoring disease activity remains uncertain [11-]. 
MRI has also been used to evaluate the cranial arteries, with a pooled sensitivity of around $73 \%$ and specificity of $88 \%$. Because of the cost and limited availability of MRI, however, as well as the possible adverse effects of contrast reagents, MRI is not recommended for first-line use in imaging [1-m].

\section{GLUCOCORTICOIDS AND THEIR TOXICITIES}

Glucocorticoids were first-line therapy - and the monotherapy - for GCA for nearly 70 years. Conventional wisdom dictated the importance of high initial doses of prednisone, typically on the order of $1 \mathrm{mg} / \mathrm{kg} / \mathrm{day}$, or pulse-dose methylprednisolone $1 \mathrm{~g}$ intravenously daily for 3 days in the setting of imminent vision impairment or frank vision loss. Treatment with glucocorticoids generally consisted of a minimum of 1 year of prednisone or another glucocorticoid, tapering from high to low doses over many months without a specified endpoint. The majority of patients treated with prednisone alone ultimately require additional dosing because of flares or refractory symptoms, particularly if attempts are made to stop glucocorticoids entirely within 1 year [12-m].

Despite this unquestioned efficacy of high-dose prednisone for quelling active GCA and preventing vision loss, the toxicities of conventional glucocorticoid dosing for the treatment of GCA are daunting. Up to $90 \%$ of patients have at least one adverse event while taking glucocorticoids, with the most frequent occurrences being cataracts and bone mineral disease. For each $1000 \mathrm{mg}$ increase in glucocorticoid exposure, the hazard ratio for adverse events increases by 3\% [13]. A series of nested case-control analyses evaluated numerous complications as well as the dose-dependent nature of the risk. Among GCA patients taking prednisone $30 \mathrm{mg}$ daily or more compared with those on lower daily prednisone $(5 \mathrm{mg}$ daily or less), the odds ratios for complications were: diabetes (4.7), osteoporosis (1.9), fractures (2.6), glaucoma (3.5), serious infection (3.3), and death (2.1), with many complications occurring after years [14].

Another study showed similar rates of osteoporosis and fractures among male and female individuals despite the fact that female individuals have more risk factors at baseline for fracture [15]. The possibility of weight gain is also a particular concern to many patients and may contribute to poor adherence. Prior studies in vasculitis have shown mixed results regarding the effect of glucocorticoids on weight gain. Improvement in disease activity, for example, is associated with weight gain regardless of cumulative prednisone dose, and patients who fail to achieve disease control may not experience weight gain despite receiving larger doses of glucocorticoids [16,17].

\section{TREATMENT WITH TOCILIZUMAB}

The first reported randomized controlled trial to assess the efficacy of tocilizumab in GCA randomized 20 patients to either tocilizumab $8 \mathrm{mg} / \mathrm{kg}$ intravenous monthly or placebo infusions in addition to glucocorticoids and found a higher relapse-free survival in the tocilizumab group (85 versus 20\%, $P=0.001$ ) at week 52 [18]. The Giant Cell Arteritis Actemra (GiACTA) trial enrolled 251 patients, randomized to one of four arms: tocilizumab $162 \mathrm{mg}$ weekly or every other week (combined with a 26-week prednisone taper), or a 
prednisone taper alone (either 26 or 52 weeks). The primary endpoint - the rate of sustained glucocorticoid-free remission at week 52 - was achieved in $56 \%$ of the weekly tocilizumab group and $53 \%$ of the every other week tocilizumab group compared with $14 \%$ in the 26 week prednisone group and $18 \%$ in the 52-week prednisone group. The cumulative prednisone dose was significantly lower in both tocilizumab groups compared with both prednisone groups. Serious adverse events were seen more frequently in the prednisone groups [12-- The effects of tocilizumab on glucocorticoid-sparing were observed in both relapsing and newly diagnosed GCA.

\section{TREATMENT WITH USTEKINUMAB}

Ustekinumab has also been studied as a potential glucocorticoid-sparing agent in GCA, but with less consistently positive results and is not Food and Drug Administration (FDA)approved for treatment. The investigators in one open-label study of 25 patients with refractory GCA treated all patients with ustekinumab in addition to glucocorticoids and demonstrated that no patients relapsed while on ustekinumab. Over 52 weeks, the median daily prednisolone dose decreased from 20 to $5 \mathrm{mg}$. In addition, CT angiography demonstrated improvement in large-vessel vasculitis in all patients [19]. However, a subsequent open-label study evaluating ustekinumab in combination with a 6-month prednisone taper was terminated early because of the observation of disease flares in 7 out of the first $11(63.6 \%)$ patients enrolled. Only two patients (18\%) achieved the primary outcome of prednisone-free remission with normal inflammatory markers at 52 weeks [20]. The fundamental difference in these two open-label experiences with ustekinumab appears to be the maintenance of glucocorticoid therapy in one, and the discontinuation of glucocorticoid treatment completely in the other.

\section{OTHER TREATMENT MODALITIES}

Abatacept, a CTLA-4 immunoglobulin that acts as a negative regulator of T-cell costimulation, was studied in a randomized withdrawal trial design. The relapse-free survival rate in the abatacept group was $48 \%$ compared with $31 \%$ in the placebo group (onesided $P$-value $=0.049$ ). There was also a longer median duration of remission in the abatacept group (9.9 versus 3.9 months) and no increase in adverse events though abatacept is not FDA approved for GCA treatment [21].

\section{HOW LONG SHOULD TOCILIZUMAB BE CONTINUED?}

Although tocilizumab has shown encouraging results as a glucocorticoid-sparing treatment for GCA, the optimal duration of treatment remains unknown. A follow-up study of 17 patients who had received 1 year of tocilizumab treatment and were in treatment-free remission at the time of tocilizumab cessation showed that eight patients (47\%) relapsed after a mean of 6.3 months. The patients in that study who relapsed following the discontinuation of tocilizumab were younger and had a greater degree of vessel wall enhancement on MRI at baseline compared with those who did not flare. All of the patients in the study, however, had persistent MRI abnormalities at follow-up [22]. The proper interpretation of persistent MRI enhancement in GCA remains uncertain. 
A long- term, 2-year extension of the GiACTA trial followed patients who had received either tocilizumab with glucocorticoids or glucocorticoids alone, with treatment at the discretion of the provider. Forty-nine percent of the patients in the weekly tocilizumab group and $39 \%$ of the patients in the every other week tocilizumab group maintained complete remission during the entirety of part 2 , and $65 \%$ of these patients were treatment free. The highest proportion of patients who maintained complete remission while not on any treatment was $68 \%$ in the weekly tocilizumab group. Forty-two percent of the patients who achieved sustained disease remissions on weekly tocilizumab and a 26-week prednisone taper maintained treatment-free remissions for 2 years after tocilizumab discontinuation, underscoring the point that although vigilance for the possibility of disease flares remains crucial in GCA, not all patients require continuous treatment with immunosuppressive medications [23-].

\section{RISK FACTORS FOR TREATMENT FAILURE OR RELAPSE}

Despite treatment with glucocorticoids and even tocilizumab, a substantial portion of patients still experience flares either during or after the discontinuation of these immunosuppressive medications. A study of 149 tocilizumab-treated patients showed that $24 \%$ experienced a flare, and $64 \%$ were still receiving prednisone at the time of the flare. Approximately $25 \%$ of the disease flares occurred while patients were taking greater than 10 $\mathrm{mg}$ daily [24]. Inflammatory markers are not reliable indicators of flare, particularly in patients receiving IL6-R blockade treatment. Ninety-two percent of patients receiving tocilizumab had normal C-reactive protein (CRP) levels at the time of flare. Moreover, 34\% of patients treated with prednisone alone had normal CRP levels at disease flare. This highlights the need for the discovery of additional biomarkers.

The study described above that reported a higher degree of mural enhancement on MRI among patients who relapsed after stopping tocilizumab treatment found no differences in sex, presence or absence of cranial symptoms, presence or absence of a positive temporal artery biopsy, or other factors that predicted posttreatment relapse [22].

A follow-up analysis from the GiACTA trial, however, showed that female sex, worse patient-reported outcomes at baseline, and treatment with prednisone alone (as opposed to tocilizumab) were independent predictors of treatment failure [25]. In a multivariate analysis, women with GCA were more than five times more likely than men to fail treatment if treated with prednisone alone. The two strongest risk factors for GCA flare were the absence of treatment with tocilizumab and female sex.

Although it has been hypothesized that patients with GCA who have involvement of the aorta and its primary branches as opposed to cranial GCA have a disease phenotype that is more difficult to treat, to date the outcomes of patients with GCA and large vessel involvement have not been proved different compared with those with cranial disease only [10]. 


\section{USE OF BIOMARKERS TO IDENTIFY FLARE}

Attempts have been made to identify biomarkers that could be useful in monitoring disease and identifying a flare. This is especially important as tocilizumab suppresses the erythrocyte sedimentation rate (ESR) and CRP, thus rendering them unreliable as markers of disease activity. Despite the role of IL-6 in GCA, IL-6 levels have not been shown to correlate with disease activity [24].

One study found that matrix metalloproteinase-3 (MMP-3), pentraxin-3, and soluble tumor necrosis factor receptor 2 (sTNFR2) were significantly elevated in GCA patients compared with age-matched and sex-matched controls, and that tocilizumab resulted in normalization of these levels. MMP-3 levels also had a weak association with MRI signal intensity on MRA of the aortic wall [26]. Two studies recently evaluated the role of serum amyloid A in evaluating disease activity. One study found that the serum amyloid A (SAA) levels were significantly higher in GCA patients with active disease compared with inactive disease [27-]. Another study analyzed the profiles of healthy blood donors and patients with GCA and found that levels of SAA as well as interleukin-23 (IL-23) and IL-6 were significantly higher in patients with GCA compared with healthy controls. Changes in the SAA levels also correlated with relapses of disease as well as visual disturbance [28]. Though these serum markers are not currently used in clinical practice, these studies may pave the way for studies of therapy targeted toward SAA.

\section{MONITORING WITH SERIAL IMAGING}

Longitudinal MRI, PET/CT, and ultrasound have been studied in GCA patients with mixed results. One study showed that all patients in lasting remission still had enhancement on MRI with low intensity vessel wall signal at the time of follow-up [22]. Another study also showed that MRI vessel wall signal does not parallel clinical disease activity [29-]. Similarly with PET/CT, the majority of patients still have some degree of vascular uptake after 1 year, though the level of maximal uptake can potentially help to distinguish patients in remission from those with active disease [11 $\mathbf{4}, 30]$. For temporal artery ultrasound, one group described two patients in whom the halo sign was significantly diminished with tocilizumab treatment, and further studies are ongoing to evaluate this [31]. The use of imaging to confirm or exclude a flare is not known though is recommended for long-term monitoring of patients with known large vessel involvement [1- $]$.

\section{CONCLUSION}

In summary, the diagnosis and treatment of GCA have evolved substantially in recent years. Temporal biopsy was once regarded as the only definitive way to diagnose GCA, whereas noninvasive imaging studies can now be used. Furthermore, some noninvasive imaging studies are also helpful for detecting large vessel or aortic involvement. Their role in the longitudinal monitoring of disease activity requires further evaluation. Similarly, long-term treatment with glucocorticoids was regarded as the only reliable therapeutic option for patients with GCA for decades. Tocilizumab has emerged recently as an agent that substantially increases the rate of glucocorticoid-free remission and reduces the cumulative 
glucocorticoid doses required to maintain disease control. Further studies are necessary to identify other novel treatment options as well as other biomarkers that can accurately identify flares.

\title{
Acknowledgements
}

\author{
Financial support and sponsorship \\ N.S.-B. receives funding from the Research Training in Rheumatology at Massachusetts General Hospital, under \\ T32AR007258. \\ Conflicts of interest \\ N.S.-B. has no conflicts of interest to report. J.H.S. has received grants from and performed consulting for Roche \\ and has received a grant from Bristol-Myers Squibb.
}

\section{REFERENCES AND RECOMMENDED READING}

Papers of particular interest, published within the annual period of review, have been highlighted as:

- of special interest

- of outstanding interest

1-. Dejaco C, Ramiro S, Duftner C, et al. EULAR recommendations for the use of imaging in large vessel vasculitis in clinical practice. Ann Rheum Dis 2018;77:636-643. [PubMed: 29358285] These recommendations, published by EULAR, discuss that in patients with a high pretest probability, a GCA diagnosis can be made with imaging alone. They recommend temporal artery ultrasound as the first line, with high-resolution MRI as a second line. They do not recommend that CT, PET CT, or conventional angiography be used for diagnosis.

2س. Rinagel M, Chatelus E, Jousse-Joulin S, et al. Diagnostic performance of temporal artery ultrasound for the diagnosis of giant cell arteritis: a systematic review and meta-analysis of the literature. Autoimmun Rev 2019; 18:56-61. [PubMed: 30408588] This was a meta-analysis that compared the sensitivity and specificity of temporal artery ultrasound to that of temporal artery biopsy and overall found sensitivity of $68 \%$ and specificity of $81 \%$ for the hypoechoic halo, or sensitivity of $78 \%$ and specificity of $79 \%$ for any ultrasonographic abnormality.

3. Germanò G, Muratore F, Cimino L, et al. Is colour duplex sonography-guided temporal artery biopsy useful in the diagnosis of giant cell arteritis? A randomized study. Rheumatol (United Kingdom) 2015; 54:400-404.

4. Karahaliou M, Vaiopoulos G, Papaspyrou S, et al. Colour duplex sonography of temporal arteries before decision for biopsy: a prospective study in 55 patients with suspected giant cell arteritis. Arthritis Res Ther 2006; 8:R116. [PubMed: 16859533]

5. Hauenstein C, Reinhard M, Geiger J, et al. Effects of early corticosteroid treatment on magnetic resonance imaging and ultrasonography findings in giant cell arteritis. Rheumatol (United Kingdom) 2012; 51:1999-2003.

6. Achkar AA, Lie JT, Hunder GG, et al. How does previous corticosteroid treatment affect the biopsy findings in giant cell (temporal) arteritis? Ann Intern Med 1994; 120:987-992. [PubMed: 8185147]

7. Narváez J, Bernad B, Roig-Vilaseca D, et al. Influence of previous corticosteroid therapy on temporal artery biopsy yield in giant cell arteritis. Semin Arthritis Rheum 2007; 37:13-19. [PubMed: 17360027]

8. Maleszewski JJ, Younge BR, Fritzlen JT, et al. Clinical and pathological evolution of giant cell arteritis: a prospective study of follow-up temporal artery biopsies in 40 treated patients. Mod Pathol 2017; 30:788-796. [PubMed: 28256573]

9๘. Sammel AM, Hsiao E, Schembri G, et al. Diagnostic accuracy of positron emission tomography/ computed tomography of the head, neck, and chest for giant cell arteritis: a prospective, double- 
blind, cross-sectional study. Arthritis Rheumatol 2019; 71:1319-1328. [PubMed: 30848549] This prospective study evaluated 64 patients with newly suspected GCA who underwent time of flight PET CT to evaluate the temporal arteries. They found that compared with temporal artery biopsy, the sensitivity of PET CT was $92 \%$ and specificity was $85 \%$, and compared with clinical diagnosis, the sensitivity was $71 \%$ and specificity was $91 \%$. Additionally, the negative predictive value of $98 \%$ indicates that it can be helpful in ruling out GCA in patients with a low pretest probability. Additionally, aortitis was seen in $42 \%$ of patients with a positive temporal artery biopsy.

10. de Boysson H, Liozon E, Lambert M, et al. Giant-cell arteritis: do we treat patients with largevessel involvement differently? Am J Med 2017; 130:992-995. [PubMed: 28460851]

11. Braun J, Baraliakos X, Fruth M. The role of 18F-FDG positron emission tomography for the diagnosis of vasculitides. Clin Exp Rheumatol 2018; 36(Suppl 114):S108-S114.This review discussed the use of PET CT for the diagnosis of vasculitis. Several of the main points were that the diagnosis is most accurate in the first 3 days after glucocorticoid administration, that supraaortic arteries provide the best identification of GCA patients. The ascending aorta and axillary arteries had the highest uptake. The value of PET CT in follow-up imaging and disease flares is less clear.

12m. Stone J, Tuckwell K, Dimonaco S, et al. Trial of tocilizumab in giant-cell arteritis. NEJM 2017; 377:317-328. [PubMed: 28745999] This landmark trial randomized 251 patients to tocilizumab (weekly or every other week) with a 26-week prednisone taper or placebo with either a 26 or 52 week prednisone taper. The primary endpoint of sustained glucocorticoid-free remission at week 52 was obtained in $56 \%$ of the weekly tocilizumab group and $53 \%$ of the every other week tocilizumab group compared with $14 \%$ in the 26 week taper prednisone group and $18 \%$ in the 52 week prednisone group. The cumulative prednisone was significantly reduced in the tocilizumab groups compared with the prednisone groups.

13. Broder MS, Sarsour K, Chang E, et al. Corticosteroid-related adverse events in patients with giant cell arteritis: a claims-based analysis. Semin Arthritis Rheum 2016; 46:246-252. [PubMed: 27378247]

14. Wilson JC, Sarsour K, Collinson N, et al. Serious adverse effects associated with glucocorticoid therapy in patients with giant cell arteritis (GCA): a nested case-control analysis. Semin Arthritis Rheum 2017; 46:819-827. [PubMed: 28040244]

15. Wilson JC, Sarsour K, Collinson N, et al. Incidence of outcomes potentially associated with corticosteroid therapy in patients with giant cell arteritis. Semin Arthritis Rheum 2017; 46:650656. [PubMed: 27839741]

16. Wallace ZS, Miloslavsky EM, Cascino M, et al. Effect of disease activity, glucocorticoid exposure, and rituximab on body composition during induction treatment of antineutrophil cytoplasmic antibody-associated vasculitis. Arthritis Care Res 2017; 69:1004-1010.

17. Wung PK, Anderson T, Fontaine KR, et al., WEGENER'S GRANULOMATOSIS ETANERCEPT TRIAL RESEARCH GROUP. Effects of glucocorticoids on weight change during the treatment of Wegener's granulomatosis. Arthritis Care Res 2008; 59:746-753.

18. Villiger PM, Adler S, Kuchen S, et al. Tocilizumab for induction and maintenance of remission in giant cell arteritis: a phase 2, randomised, double-blind, placebo-controlled trial. Lancet 2016; 387:1921-1927. [PubMed: 26952547]

19. Conway R, O’Neill L, Gallagher P, et al. Ustekinumab for refractory giant cell arteritis: a prospective 52-week trial. Semin Arthritis Rheum 2018; 48:523-528. [PubMed: 29776658]

20. Matza M, Stone J, Fernandes A, Unizony S. Ustekinumab for the treatment of giant cell arteritis (abstract 1837). American College of Rheumatology's Annual Meeting. 2019.

21. Langford C, Cuthbertson D, Ytterberg S, et al., Vasculitis Clinical Research Consortium. A randomized, double-blind trial of abatacept (CTLA-4Ig) for the treatment of giant cell arteritis. Arthritis Rheumatol 2017; 69:837-845. [PubMed: 28133925]

22. Adler S, Reichenbach S, Gloor A, et al. Risk of relapse after discontinuation of tocilizumab therapy in giant cell arteritis. Rheumatology (Oxford) 2019; 58:1639-1643. [PubMed: 30915462]

23m. Stone J, Bao M, Han J, et al. Long-term outcome of tocilizumab for patients with giant cell arteritis: results from part 2 of a randomized controlled phase 3 trial (abstract 808). American College of Rheumatology's Annual Meeting. 2019.This study was a long-term, 2-year extension 
of the GiACTA trial and showed that $49 \%$ of patients in the weekly tocilizumab group remained complete remission for the 2 years following discontinuation.

24. Stone JH, Tuckwell K, Dimonaco S, et al. Glucocorticoid dosages and acute-phase reactant levels at giant cell arteritis flare in a randomized trial of tocilizumab. Arthritis Rheumatol 2019; 71:1329-1338. [PubMed: 30835950]

25. Unizony S, Bao M, Han J, et al. Risk factors for treatment failure in patients with giant cell arteritis treated with tocilizumab plus prednisone versus prednisone alone. Ann Rheum Dis 2019; 78(Suppl 2):810 (abstract 0261).

26. Gloor AD, Yerly D, Adler S, et al. Immuno-monitoring reveals an extended subclinical disease activity in tocilizumab-treated giant cell arteritis. Rheumatology (Oxford) 2018; 57:1795-1801. [PubMed: 29961816]

27-. Dartevel A, Toussaint B, Trocme C, et al. Serum amyloid A as a marker of disease activity in Giant cell arteritis. Autoimmun Rev 2019; 19:102428. [PubMed: 31734404] This study evaluated 26 patients during the course of newly diagnosed and relapsing giant cell arteritis and found that the serum amyloid A (SAA) levels were significantly higher in active disease compared with inactive disease.

28. Burja B, Feichtinger J, Lakota K, et al. Utility of serological biomarkers for giant cell arteritis in a large cohort of treatment-naive patients. Clin Rheumatol 2019; 38:317-329. [PubMed: 30143961]

29-. Reichenbach S, Adler S, Bonel H, et al. Magnetic resonance angiography in giant cell arteritis: results of a randomized controlled trial of tocilizumab in giant cell arteritis. Rheumatology (Oxford) 2018; 57:982-986. [PubMed: 29529280] This study used data from a clinical trial of tocilizumab versus glucocorticoids and found that 19 of 33 patients initially had a positive MRA. After a year of treatment, roughly one-third of patients treated with tocilizumab still showed persistent changes on MRI, despite patients being in remission by clinical and laboratory parameters.

30. Nannini C, Niccoli L, Sestini S, et al. Remission maintenance after tocilizumab dose- tapering and interruption in patients with giant prospective, pilot study. Ann Rheum Dis 2019; 78:1444-1446. [PubMed: 31213436]

31. Evangelatos G, Fragoulis GE, Iliopoulos A. Temporal ultrasound for monitoring tocilizumab treatment in giant cell arteritis: seeing beyond serum markers? Ann Rheum Dis 2019; doi:10.1136/ annrheumdis-2019-216527. [Epub ahead of print] 


\section{KEY POINTS}

- Noninvasive imaging techniques can allow for a diagnosis of GCA without a temporal artery biopsy.

- Glucocorticoids remain a mainstay in the treatment of GCA, though are associated with numerous toxicities.

- Tocilizumab helps to reduce the rate of flare, increase remission, and reduce the cumulative dose of glucocorticoid. 


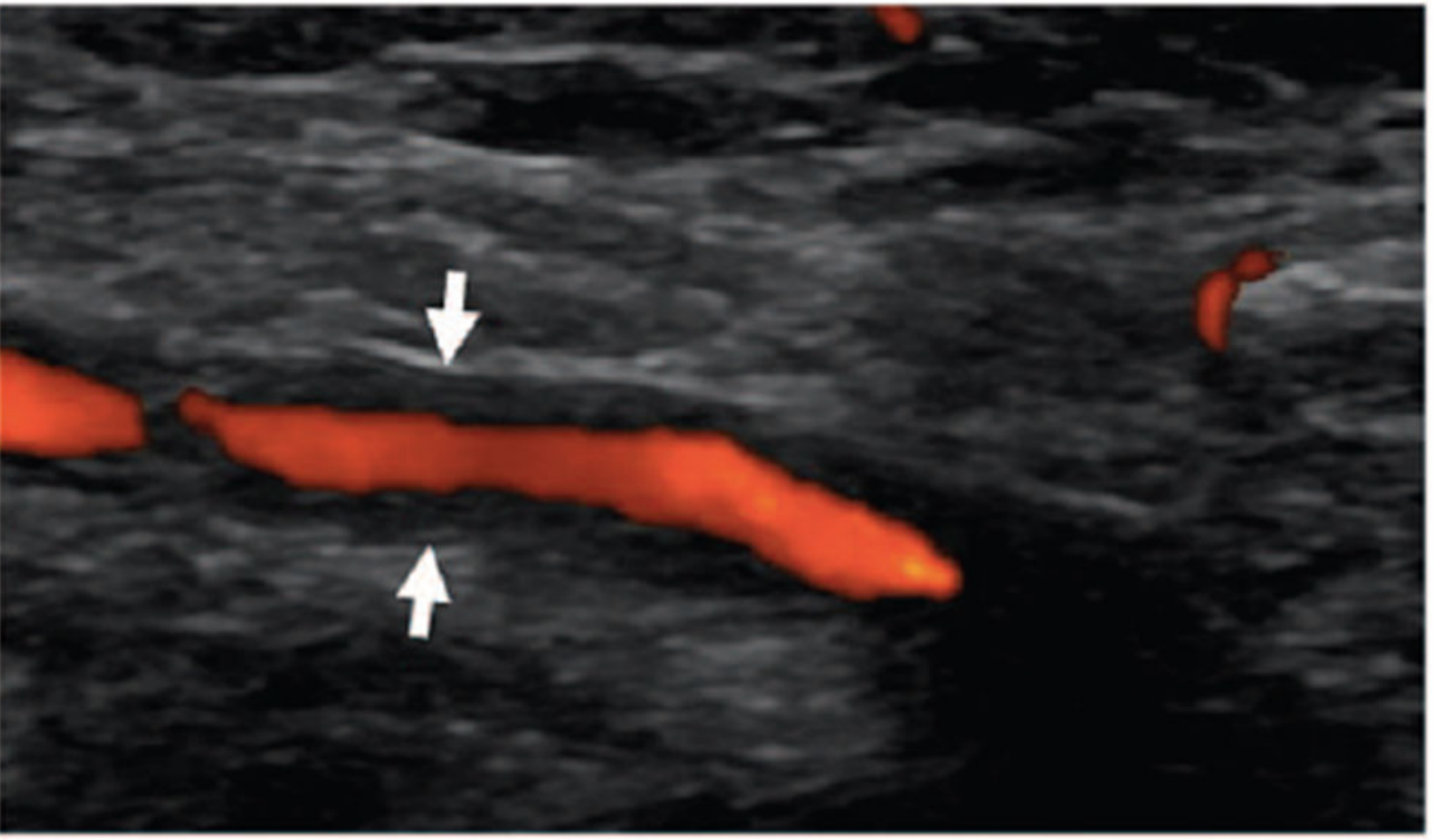

FIGURE 1.

Temporal artery ultrasound with doppler demonstrating the halo sign. Ultrasound demonstrating a longitudinal view of the left superficial cutaneous temporal artery. The halo sign is indicated by the white arrows and is demonstrated as a hypoechoic lining around the artery. 


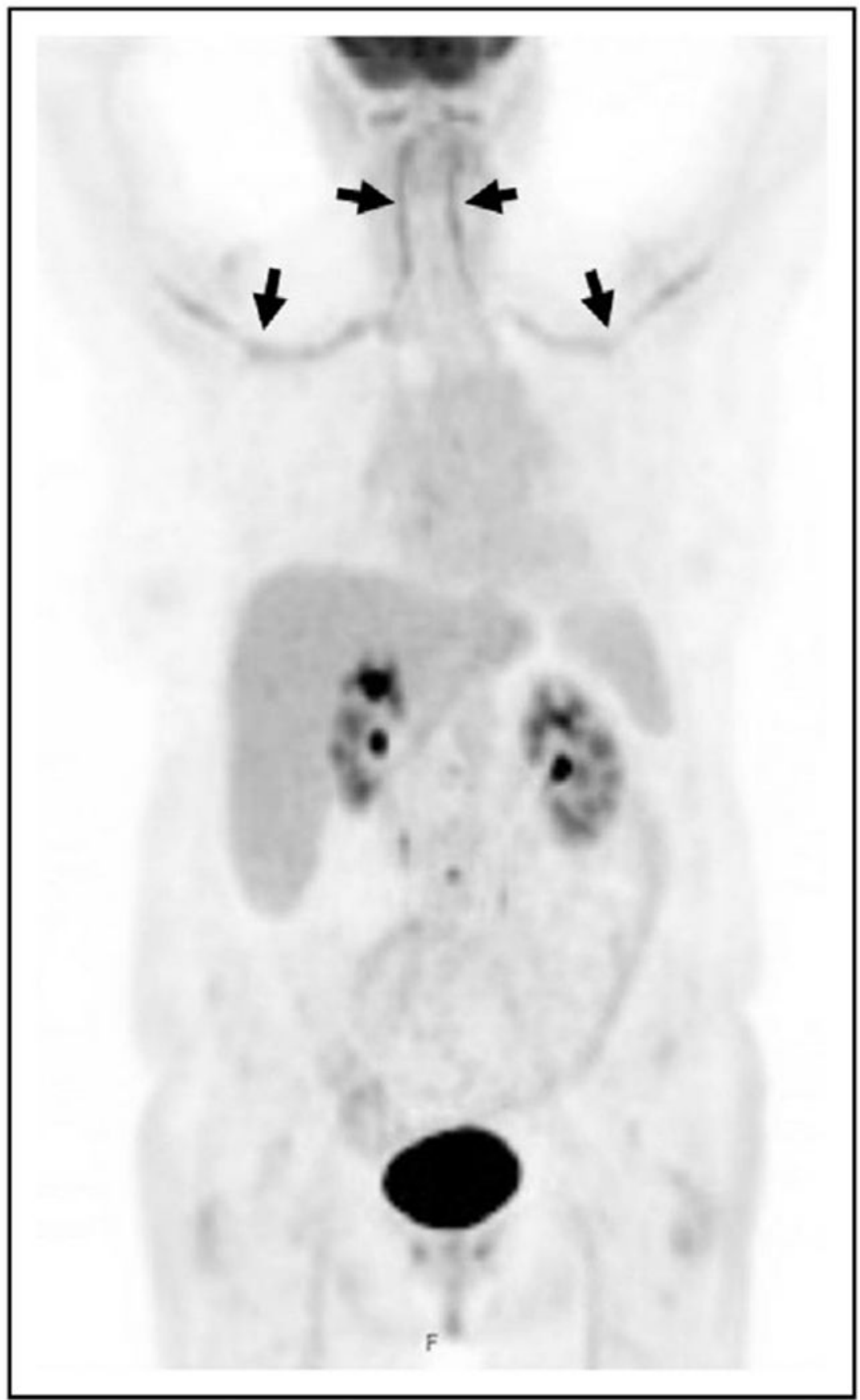

FIGURE 2.

Large vessel vasculitis on positron emission tomography/computed tomography (PET/CT). $\mathrm{PET} / \mathrm{CT}$ in a patient with giant cell arteritis demonstrating intense fluorodeoxyglucose avidity in the bilateral vertebral and subclavian arteries, indicated by the black arrows. 


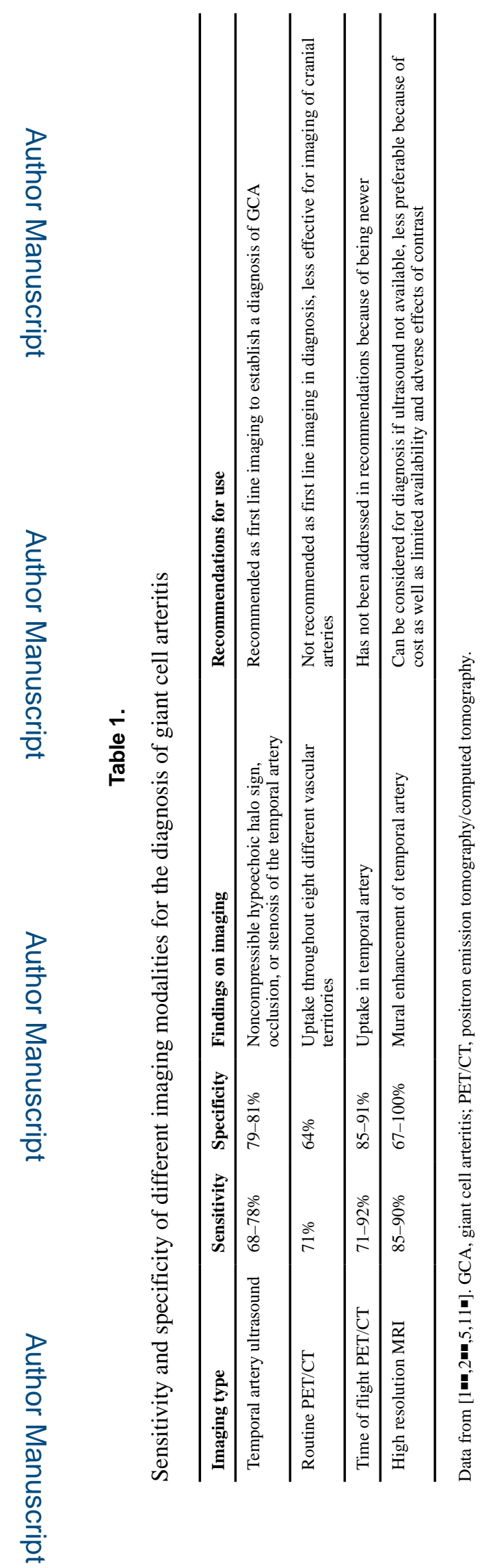

Curr Opin Rheumatol. Author manuscript; available in PMC 2020 May 15. 